\title{
Outcomes of tracheostomy in COVID-19 patients in National Guard Health Affairs, Riyadh, Saudi Arabia
}

Salwa ALHumaid, MD, MohammedA. Elkrim, MD, YazeedA. AlOqaili, MD, Ghada A. AlSowailmi, MBBS, FahadA. AlObaid, MD, Abdulaziz A. AlSalem, MD, Mohammed A. AlQabasani, MD, Yaseen M. Arabi, MD, Hasan M. AlDorzi, MD.

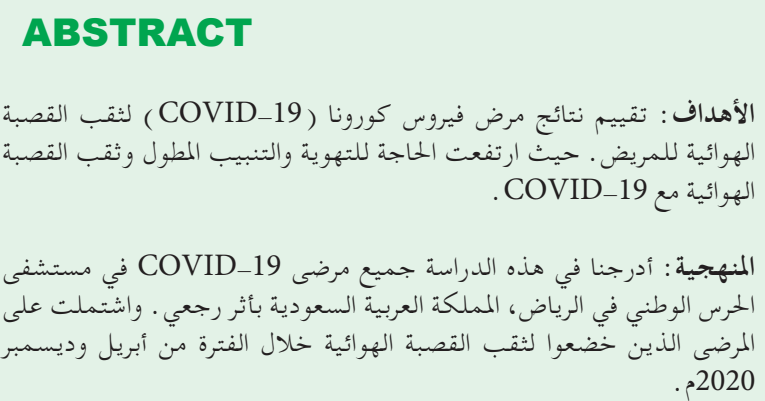

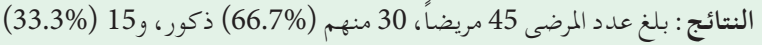

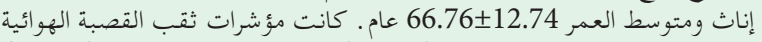

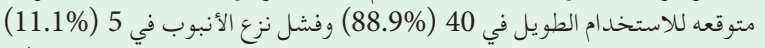

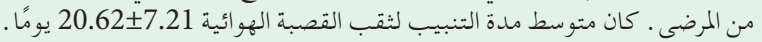

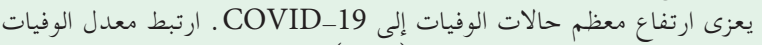

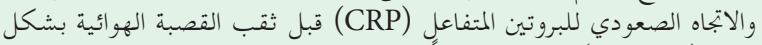

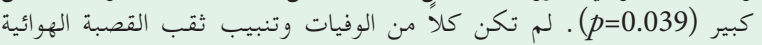

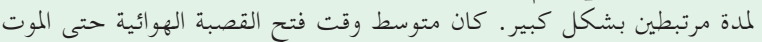

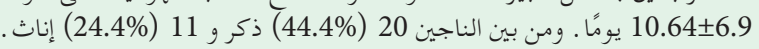

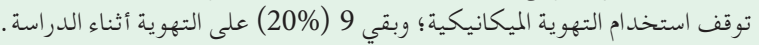

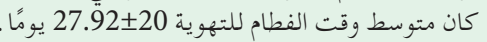

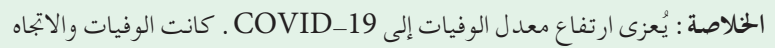

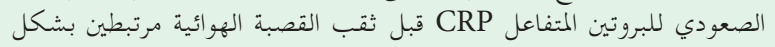

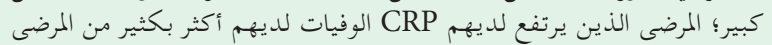

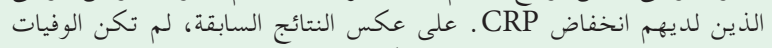
وثقب القصبة الهو ائية لمدة مرتبطين بشكل كبير.

Objectives: To evaluate coronavirus disease 2019 (COVID-19) patient tracheostomy outcomes.

Methods: All COVID-19 patients at the National Guard Hospital, Riyadh, Saudi Arabia, were retrospectively recruited. Those who had tracheostomies between April and December 2020 were included.

Results: The population was 45 patients, of which 30 (66.7\%) were males, $15(33.3 \%)$ were females and the mean age was $66.76 \pm 12.74$ years. The tracheostomy indications were anticipated prolonged weaning in $40(88.9 \%)$ and failed extubation in $5(11.1 \%)$ of the patients. The mean intubation to tracheostomy duration was 20.62 \pm 7.21 days. Mortalities were high, with most attributed to COVID-19. Mortality and a pre-tracheostomy C-reactive protein (CRP) uptrend were significantly related $(p=0.039)$. Mortality and intubation to tracheostomy duration were not significantly related. The mean post-tracheostomy time to death was $10.64 \pm 6.9$ days. Among the survivors, $20(44.4 \%)$ males and $11(24.4 \%)$ females were weaned off mechanical ventilation; 9 (20\%) remained on ventilation during the study. The mean ventilation weaning time was $27.92 \pm 20$ days.

Conclusion: The high mortality rate was attributed to COVID-19. Mortality and a pre-tracheostomy CRP uptrend were significantly related; uptrend patients experienced far more mortalities than downtrend patients. Unlike previous findings, mortality and intubation to tracheostomy duration were not significantly related.

Keywords: COVID-19, aerosol generating procedure, tracheostomy, prolonged intubation, personal protective equipment

Saudi Med J 2021; Vol. 42 (11): 1217-1222 doi: 10.15537/smj.2021.42.11.20210505

From the Division of Otolaryngology-Head and Neck Surgery (ALHumaid, Elkrim, AlOqaili, AlObaid, AlSalem, AlQabasani), Department of Surgery; from the Department of Intensive Care Unit (Arabi, AlDorzi), King Abdulaziz Medical City and King Abdullah Specialist Children's Hospital National Guard Health Affairs, and from the College of Medicine (AlSowailmi), King Saud bin Abdulaziz University for Health Sciences, Riyadh, Kingdom of Saudi Arabia.

Received 25th June 2021. Accepted 28th September 2021.

Address correspondence and reprint request to: Dr. Ghada A. Alsowailmi, College of Medicine, King Saud bin Abdulaziz University for Health Sciences, Riyadh, Kingdom of Saudi Arabia. E-mail: alsowailmi291@ksau-hs.edu.sa

ORCID ID: https://orcid.org/0000-0001-5457-0576 
C oronavirus disease 2019 (COVID-19) is an infectious disease caused by the strain severe acute respiratory syndrome coronavirus -2 (SARS-CoV-2). The virus was recently discovered in Wuhan, China, in December 2019, after which it became a pandemic that affected most countries in the world. By June 27, 2020, the Kingdom of Saudi Arabia's Ministry of National Guard Health Affairs (MNGHA) had reported 7,108 cases with 108 intensive care unit (ICU) admissions and 94 associated deaths. ${ }^{1-3}$ The number was expected to grow with an increase in ventilation, prolonged intubations and tracheostomies for patients, mostly requested by critical care professionals.

Current data shows that the prognosis for patients who have COVID-19 and have been intubated is poor; such patients have a high rate of mortality. ${ }^{3-6}$ The present data can help ensure the safety of patients and healthcare practitioners and minimize risks of hospital-acquired infections. This study aimed to evaluate these outcomes to provide a basis for tracheostomy recommendations in regards to the COVID-19 pandemic.

Methods. This is a single-center retrospective study of 45 patients, of which $30(66.7 \%)$ were males and 15 $(33.3 \%)$ were females. Data was collected and processed from patients' charts at the tertiary care facility of the National Guard Hospital, Riyadh, Kingdom of Saudi Arabia (KSA). All the hospital's COVID-19 patients who had undergone a tracheostomy at the hospital's division of otolaryngology, head and neck surgery for any reason between April 2020 and December 2020 were retrospectively included. Any patient who was not infected with COVID-19 before their tracheostomy date was excluded from the study.

This study was approved by the Research and Ethics Committee of the King Abdullah International Medical Research Center, Riyad, KSA (approval no. RC20/372/R). No consent forms were used due to the observational nature of the research, and the anonymity of the participants was preserved throughout the study. All information was kept confidential; only the research team had access to the data, which was kept in both hard and soft formats in a secure place on the MNGHA's premises. All research reporting was done per the Strengthening, the Reporting of Observational Studies in Epidemiology statement. ${ }^{7}$

The authors reviewed the patients' paper and electronic charts, then extracted data relevant to the

Disclosure. Authors have no conflict of interests, and the work was not supported or funded by any drug company. study. The data was then reviewed for accuracy by a specialist. Baseline characteristic variables were collected, including age, gender, nationality, body mass index (BMI) and comorbidities. Durations from admission to intubation and durations from intubation to tracheostomy were measured in days. Different parameters regarding tracheostomies were collected as well, including temperature, previous non-invasive ventilation (NIV) usage, inotrope usage and different ventilatory settings, including positive end-expiratory pressure (PEEP), fraction of inspired oxygen ( $\mathrm{FiO} 2)$ and C-reactive protein (CRP) uptrends and downtrends pre-operation. A CRP value of more than $8 \mathrm{mg} / \mathrm{L}$ was considered abnormal. The type of tracheostomy that was performed was noted as either open or percutaneous.

Furthermore, for outcome measurements, the following parameters were collected: intra- and postoperative complications, mortalities, causes of death, durations to deaths after tracheostomies, ventilator weaning durations, if weaned and whether patients were safely discharged from the ICU.

Statistical analysis. The data was entered into Microsoft Excel (Microsoft Corporation, Version 16.51, USA) and analyzed using Statistical Package for the Social Sciences for Windows, version 28 (IBM Corp., Armonk, NY, USA). The results were presented as descriptive statistics. The mean and standard deviation of the data was used for numerical variables and as frequencies and percentages for categorical variables; a Chi-squared test was used to compare categorical variables and Pearson correlation was used to compare numerical variables. The data was presented with a $95 \%$ confidence interval (CI), and a $p$-value $<0.05$ was considered significant.

Results. Table 1 shows a socio-demographic profile of the participants, of which $30(66.7 \%)$ were males and $15(33.3 \%)$ were females. In terms of BMI, the majority of the present study were class I obesity and overweight. The patients' minimum age was 37 years, maximum age was 97 years and mean age was $66.76 \pm 12.7$ years.

Every patient had a history of a positive COVID-19 swab preceding the operation. However, the second-last COVID-19 tests the patients received before their tracheostomies were positive for $22(48.9 \%)$ of the patients, negative for $4(8.9 \%)$ of the patients and not applicable to 19 (42.2\%) of the patients. Moreover, the outcomes of the last COVID-19 tests the patients received before their tracheostomies were positive for $39(86.7 \%)$ of the patients and negative for $6(13.3 \%)$. The mean number of repeated COVID-19 swabs was $2.16 \pm 1.4$ tests, the minimum number of swabs was 
Table 1 - The socio-demographic profiles of the participants ( $N=45)$.

\begin{tabular}{lc}
\hline Demographical characteristics & $\mathbf{n}(\%)$ \\
\hline Gender & \\
Male & $30(66.7)$ \\
Female & $15(33.3)$ \\
BMI & \\
Healthy & $2(4.40)$ \\
Overweight & $15(33.30)$ \\
Class 1 obesity & $17(37.80)$ \\
Class 2 obesity & $7(15.60)$ \\
Class 3 obesity & $4(8.90)$ \\
Age (mean \pm SD) & $66.67 \pm 12.74$ \\
\hline
\end{tabular}

BMI: body mass index, SD: standard deviation

one and the maximum number of swabs was 6 . Noninvasive ventilation was used by $30(66.7 \%)$ of the patients; accordingly, it was not used by 15 (33.3\%). Finally, $26(57.8 \%)$ of the patients were on inotropes, while $19(42.2 \%)$ were not.

Indications that a patient required a tracheostomy were anticipated prolonged weaning in $40(88.9 \%)$ of the patients and failed extubation in $5(11.1 \%)$ of the patients. Open tracheostomies were administered to $34(75.6 \%)$ of the patients, and percutaneous tracheostomies were administered to 11 (24.4\%) of the patients; the 34 open tracheostomies were carried out in operating rooms and the 11 percutaneous tracheostomies were carried out at the patients' bedsides. The mean duration from admission to intubation was $6.2 \pm 12.7$ days, the minimum duration was one day and the maximum duration was 84 days. The mean duration from intubation to tracheostomy was $20.62 \pm 7.21$ days, the minimum duration was 8 days and the maximum duration was 37 days. Moreover, the mean duration from the patients' last COVID-19 tests to their tracheostomies was $14.04 \pm 10.5$ days, with a minimum duration of one day and a maximum duration of 38 days.

The patients' mean temperature before their tracheostomies was $37.28 \pm 0.7^{\circ} \mathrm{C}$, their minimum temperature was $36.1^{\circ} \mathrm{C}$ and their maximum temperature was $39.4^{\circ} \mathrm{C}$. Their mean PEEP was $7.69 \pm 2.2$, with a minimum PEEP of 5 and a maximum PEEP of 12, while the mean $\mathrm{FiO} 2$ was $42.44 \pm 10.6$, with a $\mathrm{FiO} 2$ minimum of 25 and a maximum $\mathrm{FiO} 2$ of 75 . The CRP levels of the patients before their tracheostomies were a mean of 104.4 \pm 96.5 , a minimum CRP level of 3 and a maximum CRP level of 392.

In regards to the medical history of participants, only $4(8.9 \%)$ patients were medically free; 37 (82.2\%) had diabetes mellitus, 32 (71.1\%) had hypertension, $17(37.8 \%)$ had dyslipidemia and $9(20 \%)$ had cerebrovascular accidents. Moreover, 8 (17.78\%) had a chronic kidney disease, 7 (15.6\%) had ischemic heart disease, 6 (13.3\%) had bronchial asthma and $4(8.9 \%)$ had congestive heart failure. Finally, $3(6.7 \%)$ had atrial fibrillation, $2(4.4 \%)$ had chronic obstructive pulmonary disease and 21 (46.7\%) had other diseases.

Before the tracheostomies were administered, CRP levels uptrended in $16(35.6 \%)$ patients, downtrended in $26(57.8 \%)$ patients and did not have a trend in 3 $(6.7 \%)$ patients, the latter because only one reading was taken for each of the 3 patients before they received a tracheostomy. Only $6(13.3 \%)$ patients had postoperative complications; thus, the other $39(86.7 \%)$ patients did not. Of the complications, 2 (4.4\%) patients had a tube leakage, one $(2.2 \%)$ patient had a pneumothorax, one $(2.2 \%)$ patient had a blocked tube, one $(2.2 \%)$ patient had a displaced tube and one $(2.2 \%)$ patient had a cuff tear. It should be noted that there were zero intra-operative tracheostomy complications.

Table 2 presents the outcome details of the participants. After they received tracheostomies, 25 (55.6\%) patients died and $20(44.4 \%)$ patients were alive at the time the data was collected. The causes of death were COVID-19 related issues including cytokines storms, multiorgan failure, acute respiratory distress syndrome, or pulmonary embolism in 24 (53.3\%) patients and tracheostomy mask displacement leading to cardiorespiratory arrest in one $(2.2 \%)$ patient. The mean time to death after a tracheostomy was $10.64 \pm 6.9$ days, with a minimum time of 2 days and a maximum time of 25 days. Among the 20 (44.4\%) patients who survived, $11(24.4 \%)$ were successfully weaned off mechanical ventilation and $9(20 \%)$ were still on ventilation at time of the data was collected. The mean time to weaning after a tracheostomy was $27.92 \pm 20.21$ days, with a minimum time of 2 days and a maximum time of 82 days.

Table 2 - The outcomes of the participants $(\mathrm{N}=45)$.

\begin{tabular}{lc}
\hline Factors & $\mathbf{n}(\%)$ \\
\hline Cause of death & \\
COVID-19 related death & $24(53.3)$ \\
Tracheostomy related death & $1(2.2)$ \\
No death & $20(44.4)$ \\
Weaning from ventilator & \\
Successfully weaned from mechanical ventilation & $11(24.4)$ \\
On ventilation at the time of data collection & $9(20.0)$ \\
Death & $25(55.6)$
\end{tabular}

Duration from tracheostomy to death (in days), mean \pm SD $(\min -\max )$

$10.64 \pm 6.90(2-25)$

Duration from tracheostomy to weaning (in days)* mean $\pm S D(\min -\max )$

$27.92 \pm 20.21(2-82)$

COVID-19: coronavirus disease -19, SD: standard deviation, *: survivors 
Table 3 shows the factors associated with mortality. A significant relationship was found between mortality and a CRP uptrend before a tracheostomy $(p=0.039)$. A significantly higher mortality rate was experienced by the patients who had CRP uptrends $(75 \%)$ than by those with downtrends $(42.3 \%)$. No significant relationship was found between mortality and duration from intubation to tracheostomy, PEEP, FiO2, use of inotropes or method of tracheostomy.

Finally, Table 4 displays the factors associated with duration from tracheostomy to weaning. Method of tracheostomy was significantly related to duration from tracheostomy to weaning $(p=0.033)$. That is, a higher mean duration from tracheostomy to weaning was observed in the patients who received open tracheostomies (38.6 \pm 20 days) than in the patients who received percutaneous tracheostomies ( $15.5 \pm 12.6$ days). No significant relationship was found between duration from intubation to tracheostomy and duration from tracheostomy to weaning.

Discussion. As aforementioned, this study investigated the COVID-19 tracheostomy outcomes of patients who received assistance at a tertiary care hospital in Riyadh, Saudi Arabia. This study included various parameters that could interfere with outcome validity, including CRP, inotropes, and ventilation requirements. The majority of the study's population was male $(67 \%)$, obese $(62 \%)$, and older than 64 years (64\%); these parameters reflected findings in literature that noted a higher COVID-19 infection severity in male, obese and elderly patients. ${ }^{8}$

Indications that a patient needed a tracheostomy were anticipated prolonged ventilation $(89 \%)$ or failed extubation (11\%). Failed extubations in COVID-19 patients have been documented in extant literature and primarily attributed to laryngeal edema, which makes intubation difficult in patients who would have required straight forward intubations otherwise. ${ }^{8}$ It should be noted that the optimal duration to tracheostomy for COVID-19 patients has yet to be determined. Several guidelines have been published in this regard, but they have exhibited a wide array of recommendations. For example, the United Kingdom's guidelines recommend a tracheostomy after 14 days, while the United States of America's guidelines suggest a tracheostomy after 21 days. ${ }^{7,9}$ In the current study, the mean time from intubation to tracheostomy was 20.6 7.2 days, with a range of 8-37 days. This finding was somewhat in accordance with the results of a similar study that found an average duration to intubation of $17.5 \pm 4.9$ days and a range of 8-30 days. ${ }^{10}$

As a tracheostomy is an aerosol-generating operation that poses risks of viral transmissions to healthcare workers, the Critical Care and Acute Care Surgery Committees of the American Association for the Surgery of Trauma have recommended against administering tracheostomies to active COVID-19 patients. However, a study that examined tracheostomies in 53 patients found that no healthcare workers involved in the procedures were infected; it also found that early tracheostomies were associated with earlier ventilation weaning and recovery times. ${ }^{10}$ The fact that earlier tracheostomies may result in earlier ventilation weaning and recovery times is crucial as it can provide an advantage to those who are infected; it should therefore

Table 3 - The factors associated with patients' mortalities.

\begin{tabular}{|c|c|c|c|}
\hline \multirow[t]{2}{*}{ Factors } & Mortalities & Survivals & $P$-value* \\
\hline & \multicolumn{2}{|c|}{ Mean \pm SD } & \\
\hline Duration from intubation to tracheostomy (in days) & $20.96 \pm 7.86$ & $20.20 \pm 6.49$ & 0.730 \\
\hline PEEP & $7.36 \pm 2.06$ & $8.1 \pm 2.4$ & 0.272 \\
\hline $\mathrm{FiO} 2$ & $42.2 \pm 11.28$ & $42.75 \pm 10.06$ & 0.865 \\
\hline CRP before tracheostomy & $115.72 \pm 88.74$ & $90.15 \pm 106.03$ & 0.383 \\
\hline \multicolumn{4}{|l|}{ Use of inotropes, $n(\%)$} \\
\hline $\begin{array}{l}\text { Yes } \\
\text { No }\end{array}$ & $\begin{array}{c}16(61.5) \\
9(47.4)\end{array}$ & $\begin{array}{l}10(38.5) \\
10(52.6)\end{array}$ & 0.345 \\
\hline \multicolumn{4}{|l|}{ CRP trend before tracheostomy, $n$ (\%) } \\
\hline $\begin{array}{l}\text { Uptrend } \\
\text { Downtrend }\end{array}$ & $\begin{array}{c}12(75) \\
11(42.3)\end{array}$ & $\begin{array}{c}4(25) \\
15(57.7)\end{array}$ & $0.039^{*}$ \\
\hline \multicolumn{4}{|l|}{ Method of tracheostomy, $n$ (\%) } \\
\hline $\begin{array}{l}\text { Open } \\
\text { Percutaneous } \\
\end{array}$ & $\begin{array}{l}21(61.8) \\
4(36.4) \\
\end{array}$ & $\begin{array}{l}13(38.2) \\
7(63.6)\end{array}$ & 0.141 \\
\hline
\end{tabular}

PEEP: positive end-expiratory pressure, $\mathrm{FiO} 2$ : fraction of inspired oxygen CRP: C-reactive protein, SD: standard deviation, * significant at $<0.05$ 
Table 4 - The factors associated with duration from tracheostomy to weaning.

\begin{tabular}{lcc}
\hline Factors & $\begin{array}{c}\text { Time from tracheostomy to weaning } \\
\text { Mean } \pm \text { SD }\end{array}$ & $\begin{array}{c}\text { Pearson correlation } \\
P \text {-value }\end{array}$ \\
\hline Method of tracheostomy & $38.57 \pm 19.97$ & $0.033^{*}$ \\
$\quad \begin{array}{l}\text { Open } \\
\text { Percutaneous }\end{array}$ & $15.50 \pm 12.57$ & 0.84 \\
$\begin{array}{l}\text { Correlation between duration from intubation to } \\
\text { tracheostomy and duration from tracheostomy } \\
\text { to weaning }\end{array}$ & -0.63 \\
\hline \multicolumn{2}{c}{ SD: standard deviation, * : significant at $<0.05$} \\
\hline
\end{tabular}

be considered a priority, especially as the pandemic has resulted in sparse resources.

However, the present study failed to confirm the correlation as there was no significant association between duration from intubation to tracheostomy and duration from tracheostomy to weaning. The only parameter that was associated with an earlier weaning time was the use of a percutaneous tracheostomy ( $p=0.033$ ) instead of an open tracheostomy. This significant relationship could be explained by the fact that a percutaneous tracheostomy requires a favorable neck anatomy, more common in non-obese patients who happen to be less severely affected by COVID-19 than in obese patients. ${ }^{8}$

A study of 87 tracheostomized patients did find that only 7 died after receiving a tracheostomy. ${ }^{11}$ However, the present study had a higher rate of mortality as 25 patients died after receiving a tracheostomy; COVID-19 related issues caused the majority of the cases. Moreover, the present study showed that an uptrending CRP level was the only factor significantly associated with mortality. This finding was in agreement with another study that concluded that both CRP and PEEP levels were independently associated with mortality in tracheostomized patients. ${ }^{11}$

Study limitations. The study population comprised patients from a single hospital in Riyadh, Saudi Arabia. The study was also limited by the small number of patients, who were included in the research over a short period of time. However, this study included all patients' comorbidities, laboratory markers and ventilatory requirements during the data collection.

In conclusion, the mortality rate in the present study was high, with the majority of mortalities attributed to COVID-19 infections. A significant relationship was found between mortalities and CRP uptrends that occurred before patients received tracheostomies; that is, a significantly higher mortality rate was experienced by the patients with CRP uptrends than by those with CRP downtrends. However, contrary to some extant literature, no significant relationship was found between mortality and duration from intubation to tracheostomy. Further randomized controlled trials are needed to investigate these associations.

Acknowledgment. The authors gratefully acknowledge the residents and nurses who helped facilitate the study. The authors would also like to thank the Department of Otorhinolaryngology, King Abdulaziz Medical City, Riyadh, Kingdom of Saudi Arabia, for their support. The authors would also like to thank Scribendi for English language editing.

\section{References}

1. World Health Organization. Weekly epidemiological update 10 November 2020. [ Updated 2020; accessed 2021 May 4]. Available from: https://www.who.int/publications/m/item/ weekly-epidemiological-update---10-november-2020

2. Saudi Center for Disease Prevention and Control. COVID-19 disease interactive dashboard. [Updated 2020; accessed 2021 May 4]. Available from: https://covid19.cdc.gov.sa/dailyupdates/

3. Michetti CP, Burlew CC, Bulger EM, Davis KA, Spain DA. Performing tracheostomy during the COVID-19 pandemic: guidance and recommendations from the critical care and acute care surgery committees of the American association for the surgery of trauma. Trauma Surg Acute Care Open 2020; 5: $\mathrm{e} 000482$.

4. Chao TN, Braslow BM, Martin ND, Chalian AA, Atkins J, Haas AR, et al. Tracheotomy in ventilated patients with COVID-19. Ann Surg 2020; 272: e30-e32.

5. Yang X, Yu Y, Xu J, Shu H, Xia J, Liu H, et al. Clinical course and outcomes of critically ill patients with SARS-CoV-2 pneumonia in Wuhan, China: a single-centered, retrospective, observational study. Lancet Respir Med 2020; 8: 475-481.

6. Zhou F, Yu T, Du R, Fan G, Liu Y, Liu Z, et al. Clinical course and risk factors for mortality of adult inpatients with COVID-19 in Wuhan, China: a retrospective cohort study. Lancet 2020; 395: 1054-1062.

7. Von Elm E, Altman DG, Egger M, Pocock SJ, Gøtzsche PC, Vandenbroucke JP. The strengthening the reporting of observational studies in epidemiology (STROBE) statement: guidelines for reporting observational studies. PLoS Med 2007; 4: e296. 
8. Li X, Xu S, Yu M, Wang K, Tao Y, Zhou Y, et al. Risk factors for severity and mortality in adult COVID-19 inpatients in Wuhan. J Allergy Clin Immunol 2020; 146: 110-118.

9. British Laryngological Association. Tracheostomy guideline COVID 19. London: BLA; [Updated 2020; accessed 2021 May 4]. Available from: https://www.britishlaryngological.org/news/ tracheostomy-guideline-covid-19
10. Chao TN, Harbison SP, Braslow BM, Hutchinson CT, Rajasekaran K, Go BC, et al. Outcomes after tracheostomy in COVID-19 patients. Ann Surg 2020; 272: e181-e186.

11. Takhar A, Surda P, Ahmad I, Amin N, Arora A, Camporota L, et al. Timing of tracheostomy for prolonged respiratory wean in critically ill coronavirus disease 2019 patients: a machine learning approach. Crit Care Explor 2020; 2: e0279. 